\title{
Voltage mode stabilisation in power systems with dynamic loads
}

\author{
M. J. Hossain ${ }^{\mathrm{a}, *}$, H. R. Pota ${ }^{\mathrm{a}}$, V. A. Ugrinovskiia ${ }^{\mathrm{a}}$, R. A. Ramos ${ }^{\mathrm{b}}$ \\ ${ }^{a}$ School of Engineering and IT, UNSW@ADFA, Northcott Drive, Canberra, ACT-2600, Australia. \\ ${ }^{b}$ Dept. of Electrical Engg., Engineering School of São Carlos, Av. Trabalhador Sãocarlense, 400 \\ 13566-590-São Carlos-SP, Brazil.
}

\begin{abstract}
This paper presents a novel modelling and excitation control design to enhance large-disturbance voltage stability in power systems with significant induction motor (IM) loads. The excitation controller is designed using minimax linear quadratic Gaussian (LQG) controller synthesis method. The nonlinear power system model is reformulated with a linear and a nonlinear term. The nonlinear term is the Cauchy remainder in the Taylor series expansion and its bound is used, in this paper, in a robust control design. An advantage of this approach over the existing linearisation scheme is the treatment of the nonlinear dynamic load model in a rigorous framework for excitation control design. The performance of the designed controller is demonstrated by large disturbance simulations on a benchmark power system for various types of loads.
\end{abstract}

Keywords: Dynamic voltage stability, Robust excitation control, Nonlinearity, Linearisation, Dynamic load, Minimax LQG Control.

\footnotetext{
${ }^{*}$ Corresponding author, Phone: +61432155461, Fax:+61 262688443.

Email addresses: m.hossain@adfa.edu.au (M. J. Hossain), h.pota@adfa.edu.au (H. R. Pota), v. ougrinovski@adfa.edu.au (V. A. Ugrinovskii), ramos@sel.eesc.usp.br (R. A. Ramos) 


\section{Introduction}

The assessment of voltage stability in power systems has recently gained increasing attention because voltage instability has been responsible for several major network collapses [1]. In contrast to the traditional machine instability problem, which deals with the rotor dynamics, voltage instability is closely related to the load behaviour [2]. Different studies have shown the importance of load representation in voltage stability analyses [1], [3]. Currently, although static load models are commonly used in the power industry to model dynamic behaviours of reactive loads, they do not adequately do so [4]. Induction machines play a crucial role in voltage dynamics [5], [6]. Motor behaviour has been a major contributing factor in a number of documented voltage instability problems and collapses [7]. The problem of voltage instability is likely to increase because of the growing use of dynamic motor loads for air conditioning, heat pumps, refrigeration, etc., [8]. Therefore, dynamic load models are needed in order to analyse voltage instability and to design controllers for enhancing stability.

The control of voltage levels is accomplished by controlling the production, absorption and flow of reactive power at all levels in a power system. There are two basic approaches to controlling voltage. One is to adjust the voltage at the generating plants by the use of excitation controllers. However, when the transmission lines are long and the generators are far away from major load centres, this method is not effective in improving voltage at the load end [2]. Still there are many situations in which excitation controllers can be used to stabilise the voltage in modern distributed power systems.

High-gain voltage regulators are often used but they deteriorate the small-signal stability of a system by reducing damping of the electromechanical modes of oscillation [9]. In this case, power system stabilisers (PSSs), supplementary to the main excitation control loop, are required to restore the small-signal stability. Another approach is to use flexible AC transmission system (FACTS) devices which act directly over the power flowing across the transmission lines in a system. However, these devices are quite expensive [10] and, therefore, where possible, the use of generator excitation control for enhancement of voltage stability is much more desirable.

A power system is highly nonlinear and a mathematical model of it obtained via linearisation may only satisfy the security requirement under small disturbances. Small disturbance 
voltage stability can be effectively studied with steady-state approaches that use linearisation of the system dynamic equation at a given operating point. However, large-disturbance voltage stability is closely related to the nonlinear behaviour of power systems [9] and controller designed using a linear model cannot ensure stability during severe disturbances. Therefore, it would be desirable to have a robust controller that could ensure the stability of a system for a wider operating region, under operating conditions that could be much more distant from the equilibrium point than the ones given by the conventional linearisation method. With this motivation, a detailed investigation into the need to include higher-order terms for small signal (modal) analysis in power systems is recently conducted by an IEEE Task Force [11]. It is shown in [12] that the addition of higher-order terms accurately predict system behaviour for large system perturbations.

Conventional excitation controllers are usually designed based on approximately linearised models dependent on a given operating condition and, therefore, may not work properly when the operating conditions change [13]. A linear optimal excitation controller based on the linear quadratic regulator (LQR ) theory has been developed to improve power system stability [14]. It is more effective, with better damping for the enhancement of power system stability, under small disturbances than with a conventional excitation controller. But this approach is often difficult to implement since not all the state variables are directly measurable. To overcome this difficulty, an output feedback LQG excitation control scheme is proposed in [15].

The LQG controller is more realistic because it is designed using only the measurable outputs and the state variables estimated from them. It is known that it provides good robustness in terms of gain margin and phase margin [16]. However, LQR and LQG controllers are unable to provide robustness against uncertainties in the operating conditions [17]. Robust LQG controllers to damp power system oscillations for thyristor-controlled series capacitors (TCSCs) are proposed in [18] and [19] in which Loop transfer recovery (LTR) method is used to design them. Robust excitation control for large uncertain power systems using the $H_{\infty}$ method is proposed in [20]. Controllers in [19] and [20], designed using linearised models, are only able to damp the oscillations due to electromechanical modes.

A feedback linearising controller (FBLC) is used in the design of a controller for a synchronous generator connected to an infinite bus in [21]. The direct feedback linearisation 
(DFBL) theory is easier to understand for power engineers but, so far, its application has been limited to single-input single-output systems. Another type of FBLC to damp electromechanical oscillations in power systems is proposed in [22], but this is achieved at the expense of reducing the voltage regulation ability of the excitation system. In [23], it is indicated that the implementation of an exciter control based on the FBLC theory requires a fixed and known equilibrium point of the power system which is usually not well-defined in practice. Furthermore, the controllers designed using a FBLC require information about the power system topology and the states must be measurable. In practice, it is very difficult to measure all states of a power system. In addition, feedback linearisation schemes need exact plant parameters to cancel the inherent system nonlinearities; this make a stability analysis an involved task. Although many developments have been proposed in the area of feedback linearisation theory, it has not yet been applied to a system with dynamic loads.

A great deal of attention has been given to the control of power systems using recently developed nonlinear control design tools to improve transient stability [24], [25], [26]. Rather than using a linearised model, as in the design of the usual PSS, nonlinear models are used and nonlinear feedback linearisation techniques are employed to linearise them which has the advantage that they are not dependent on the operating conditions as linearised models. Using nonlinear controllers, power system transient stability can be improved significantly. However, nonlinear controllers usually have a more complicated structure and are harder to implement in practice.

The design of linear controllers to enhance the dynamic stability of interconnected nonlinear power systems under different operating conditions is still a challenging task [27]. In this dissertation, we consider the problem of designing a linear controller, for a nonlinear power system model, in such a way that it performs acceptably over a wider operating region than do other conventional linear controllers. The nonlinearities are dealt with by explicitly including information about the system nonlinearities in the design formulation, using the Cauchy remainder of the Taylor series expansion.

A similar procedure has been used in [28] to design excitation controllers for large disturbances with large induction motor loads. In this paper, however, the control design algorithm is quite different compared to that in [28]. Here, the controller is designed on the faulted 
trajectory manifold which is generated by the stable equilibrium point and the controlling unstable equilibrium point. We have also used a different technique for choosing certain parameters in the design proposed in this paper (compared to the procedure for choosing them in [28]), which enabled us to significantly reduce the computational burden, as will be explained later.

The minimax LQG method [29] is used to design a robust excitation controller to stabilise the system against large disturbances using dynamic loads. Within the minimax optimal control design framework, robustness is achieved via optimisation of the worst-case quadratic performance of the underlying uncertain system. This helps to achieve an acceptable trade-off between the system's control performance and robustness.

The organisation of the paper is as follows: Section 2 provides the mathematical modelling of the power system devices under consideration. The test system and control task are presented in Section 3. Section 4 describes the linearisation technique and the technique to obtain a bound for uncertainties; Section 5 contains the essential details of the the minimax LQG controller design technique as applied to our problem; in Section 6, the designed control algorithm and performance of the controller are outlined and Section 7 presents the conclusions.

\section{Power system model}

Power system operation can be modelled at several different levels of complexity, depending on the intended application of the model. In this design, a benchmark power system model, with a large IM, is considered due to its suitability for voltage stability analysis [6]. Dynamic models used for the power system devices are presented next.

\subsection{Synchronous generator}

The actual transient behaviour of a synchronous generator in a real power system is very complex and difficult to accurately model unless some simplifications are made [30]. It is pointed out in [30] that the classical third-order single-axis generator dynamic model can be reliably used when designing an excitation controller as the dynamic phenomena with much smaller time constants (e.g., the effects of the damper windings) or much larger ones (e.g., the governor dynamics) can be neglected. The transmission network is considered as a passive 
circuit and modelled by a set of algebraic constraints representing interconnections among the dynamic models of generators and IMs. Under typical assumptions, the synchronous generator can be modelled by the following set of nonlinear differential equations [31]:

$$
\begin{aligned}
\dot{\delta} & =\omega \omega_{s}-\omega_{s}, \\
\dot{\omega} & =\frac{1}{2 H}\left[P_{m}-E_{q}^{\prime} I_{q}-D \omega\right], \\
\dot{E}_{q}^{\prime} & =\frac{1}{T_{d 0}^{\prime}}\left[E_{\mathrm{fd}}-E_{q}^{\prime}-\left(X_{d}-X_{d}^{\prime}\right) I_{d}\right],
\end{aligned}
$$

where $E_{\mathrm{fd}}$ is the equivalent emf in the exciter coil, $\delta$ is the power angle of the generator, $\omega$ is the rotor speed with respect to a synchronous reference, $E_{q}^{\prime}$ is the transient emf due to field flux linkage, $\omega_{s}$ is the absolute value of the synchronous speed in radians per second, $H$ is the inertia constant of the generator, $D$ is the damping constant of the generator, $T_{d o}^{\prime}$ is the direct-axis open-circuit transient time constant of the generator, $X_{d}$ is the synchronous reactance, $X_{d}^{\prime}$ is the transient reactance, $I_{d}$ and $I_{q}$ are direct and quadrature axis components of stator current, respectively. The mechanical input power $P_{m}$ to the generator is assumed to be constant.

\subsection{Excitation system}

The excitation system is a high gain static system and terminal voltage is measured using a transducer with first-order dynamics:

$$
\begin{aligned}
& E_{\mathrm{fd}}=K_{a} V_{c}, \\
& \dot{V}_{t r}=\frac{1}{T_{r}}\left[-V_{t r}+V_{t}\right],
\end{aligned}
$$

where $V_{t r}$ and $T_{r}$ are the output and time constant of the voltage transducer, $K_{a}$ is the gain

of the exciter amplifier, $V_{t}=\left[\left(E_{q}^{\prime}-X_{d}^{\prime} I_{d}\right)^{2}+\left(X_{d}^{\prime} I_{q}\right)^{2}\right]^{\frac{1}{2}}$, is the generator terminal voltage and $V_{c}$ is the input to the exciter (output of the designed controller).

The output-feedback controller, shown in Fig. 1, is represented as:

$$
\dot{\hat{x}}_{c}=A_{c} \hat{x}_{c}(t)+B_{c} V_{t r}, \quad V_{c}=C_{c} \hat{x}_{c}(t)
$$

where $A_{c}, B_{c}$ and $C_{c}$ are the appropriate matrices of the controller in (32)-(33). 


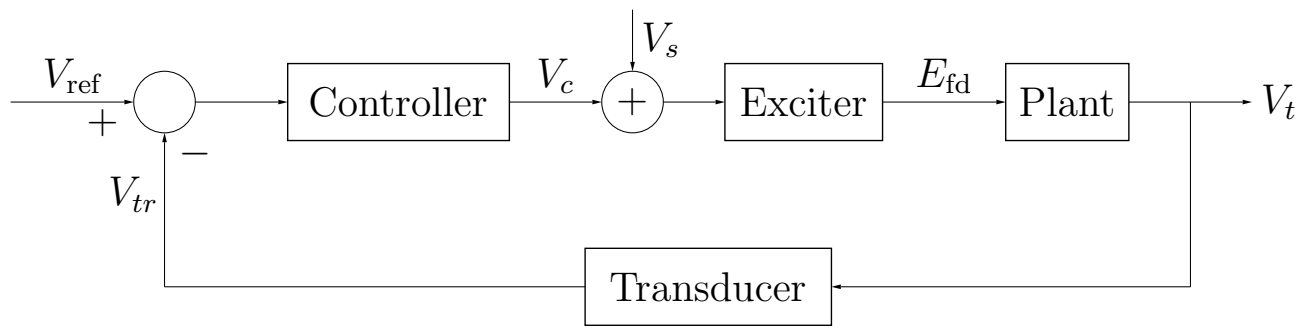

Figure 1: Excitation Controller

\subsection{Induction machine}

For representation in power system stability studies, the transient in stator voltage relations can be neglected [1], which corresponds to ignoring the DC component in stator transient currents, permitting representation of only fundamental frequency components. The transient model of an IM is described by the following algebraic-differential equations written in a synchronously-rotating reference frame [1]:

$$
\begin{aligned}
\dot{S} & =\frac{1}{2 H_{m}}\left[T_{e}-T_{L}\right], \\
T_{d o m}^{\prime} \dot{e}_{q m}^{\prime} & =-e_{q m}^{\prime}+\left(X-X^{\prime}\right) i_{d m}-T_{d o m}^{\prime} S \omega_{s} e_{d m}^{\prime}, \\
T_{d o m}^{\prime} \dot{e}_{d m}^{\prime} & =-e_{d m}^{\prime}-\left(X-X^{\prime}\right) i_{q m}+T_{d o m}^{\prime} S \omega_{s} e_{q m}^{\prime}, \\
\left(v_{d s}+j v_{q s}\right) & =\left(R_{s}+j X^{\prime}\right)\left(i_{d m}+j i_{q m}\right)+j\left(e_{q m}^{\prime}-j e_{d m}^{\prime}\right),
\end{aligned}
$$

where $X^{\prime}=X_{s}+X_{m} X_{r} /\left(X_{m}+X_{r}\right)$, is the transient reactance, $X=X_{s}+X_{m}$, is the rotor open-circuit reactance, $T_{d o m}^{\prime}=\left(L_{r}+L_{m}\right) / R_{r}$, is the transient open-circuit time constant, $T_{e}=e_{q m}^{\prime} i_{q m}+e_{d m}^{\prime} i_{d m}$, is the electrical torque, $S$ is the slip, $e_{d m}^{\prime}$ is the direct-axis transient voltage, $e_{q m}^{\prime}$ is the quadrature-axis transient voltages, $T_{L}$ is the load torque, $X_{s}$ is the stator reactance, $X_{m}$ is the magnetizing reactance, $R_{s}$ is the stator resistance, $H_{m}$ is the inertia constant of the motor, $v_{d s}$ is the $d$-axis stator voltage, $v_{q s}$ is the $q$-axis stator voltage, $i_{d m}$ and $i_{q m}$ the $d$ - and $q$-axis components of stator current, respectively. However, the above equations represent the induction machine in its own direct and quadrature axes, which are different from the $d$ and $q$ axes of the generator. A transformation is used to represent both dynamic elements with respect to the same reference frame (Appendix A). Then, the modified third-order induction machine model can be rewritten as:

$$
\left(V_{d}+j V_{q}\right)=-\left(R_{s}+j X^{\prime}\right)\left(I_{d m}+j I_{q m}\right)+j E_{q m}^{\prime},
$$




$$
\begin{aligned}
\dot{S} & =\frac{1}{2 H_{m}}\left[T_{m}-E_{m}^{\prime} I_{q m}\right] \\
\dot{E}_{m}^{\prime} & =-\frac{1}{T_{d o m}^{\prime}}\left[E_{m}^{\prime}+\left(X-X^{\prime}\right) I_{d m}\right], \\
\dot{\delta}_{m} & =S \omega_{s}-\omega_{s}-\frac{X-X^{\prime}}{T_{d o m}^{\prime} E_{m}^{\prime}} I_{q m} .
\end{aligned}
$$

To complete the model, the $d$ - and $q$-axis components of currents for both the generator and motor are given by the following network interface equations:

$$
\begin{gathered}
I_{d}=-\left(E_{q}^{\prime} B_{11}-V_{\infty}\left(G_{13} \sin \delta-B_{13} \cos \delta\right)-E_{m}^{\prime}\left(G_{12} \sin \left(\delta_{m}-\delta\right)-B_{12} \cos \left(\delta_{m}-\delta\right)\right)\right) \\
I_{q}=E_{q}^{\prime} G_{11}+V_{\infty}\left(G_{13} \cos \delta+B_{13} \sin \delta\right)+E_{m}^{\prime}\left(G_{12} \cos \left(\delta_{m}-\delta\right)-B_{12} \sin \left(\delta_{m}-\delta\right)\right) \\
I_{d m}=-\left(E_{m}^{\prime} B_{22}-V_{\infty}\left(G_{23} \sin \delta_{m}-B_{23} \cos \delta_{m}\right)-E_{q}^{\prime}\left(G_{21} \sin \left(\delta-\delta_{m}\right)-B_{21} \cos \left(\delta-\delta_{m}\right)\right)\right) \\
I_{q m}=E_{m}^{\prime} G_{22}+V_{\infty}\left(G_{23} \cos _{m}+B_{23} \sin \delta_{m}\right)+E_{q}^{\prime}\left(G_{21} \cos \left(\delta-\delta_{m}\right)-B_{23} \sin \left(\delta-\delta_{m}\right)\right)
\end{gathered}
$$

where parameters $G_{i j}$ and $B_{i j}$ are the real and imaginary parts of the equivalent transfer impedances of the reduced network between $i^{\text {th }}$ and $j^{\text {th }}$ bus. The complete model is given by equations (1)-(7) with direct and quadrature axis currents given by equations (8)-(11).

\section{Test system and control task}

The test system used in this chapter is made up of a long transmission line connecting an infinite bus to a bus with a synchronous machine and a load $\left(P_{L}=1500 \mathrm{MW}, Q_{L}=150\right.$ MVAr) [6]. Most of this load power is supplied by the remote system $(P=1200 \mathrm{MW}$, $Q=185$ MVAr) through a long double-circuit transmission line, while the remaining power (approximately $P=300 \mathrm{MW}, Q=225 \mathrm{MVAr}$ ) is supplied by the local generator at bus 1 . The nominal transmission voltage is $380 \mathrm{kV}$ and the length of the transmission line between buses 3 and 4 is $534 \mathrm{~km}$ ( twice the length of the same line given in [6]).

The single-line diagram of the test system is shown in Fig. 2 and the numerical values of the parameters are given in Appendix-B. Bus 2 represents a distribution feeder. The power consumed at this bus may correspond to a large number of individual loads fed through medium-voltage (MV) distribution lines, shunt capacitors, etc. The load at bus 2 is made up of three parts: (i) a constant impedance load; (ii) an equivalent IM and (iii) a 250 MVAr shunt capacitor for compensation purposes. The load selection is such that $50 \%$ is static and 
$50 \%$ dynamic (IM). With these load values, the operating point for the test system is: $\delta_{0}=$ $19.788^{0}, \omega_{0}=1 \mathrm{pu}, E_{q 0}^{\prime}=1.1672 \mathrm{pu}, S_{0}=0.05 \mathrm{pu}, E_{m 0}^{\prime}=0.758 \mathrm{pu}, \delta_{m 0}=22.38^{0}, V_{t r 0}=1 \mathrm{pu}$.

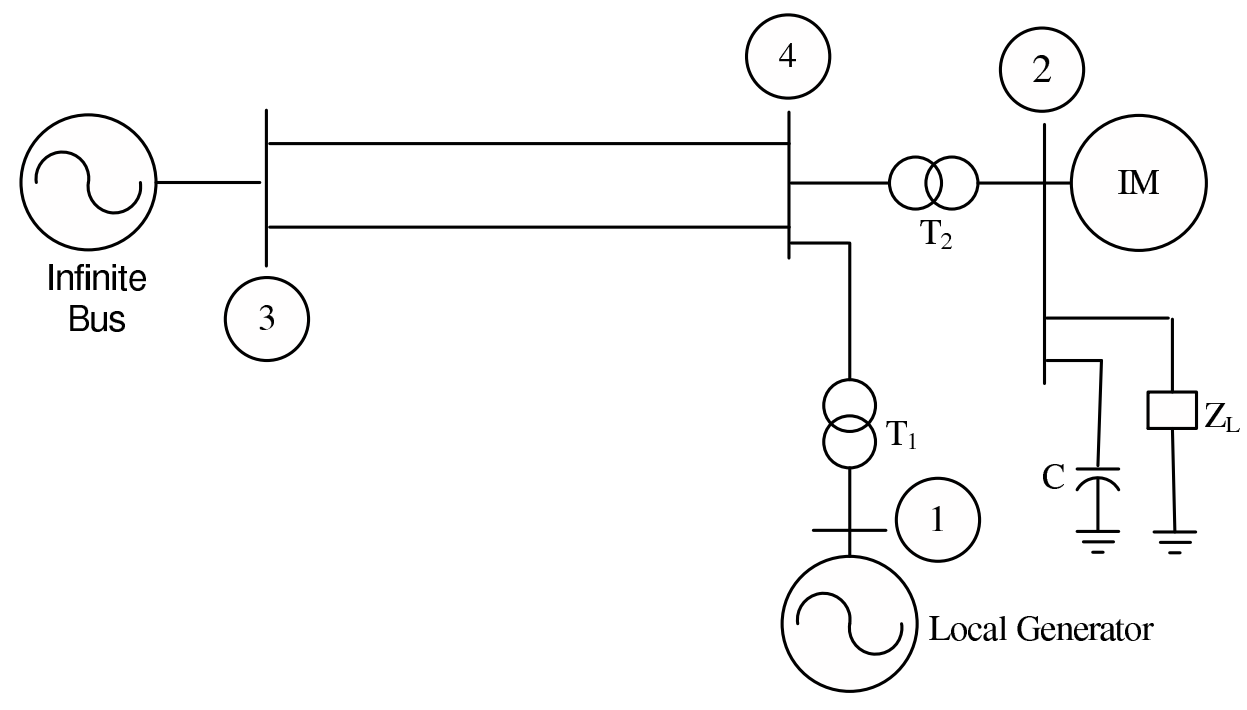

Figure 2: Test system

PSSs are designed to damp electromechanical oscillations due to large generator inertia and low damping. The objective in PSS design is to increase damping of the electromechanical mode by adding an auxiliary signal to the AVR. PSSs are designed to have very low gains in the frequency range outside of a narrow band centred around the resonant mode frequency. This necessitates the design of controllers to maintain system stability for other unstable or lightly damped modes.

The control problem considered in this case is different from the problem considered in the design of a PSS [9]. The control objective here is to stabilise unstable voltage modes which lead to voltage instability during large disturbances. The test system in this chapter, with a $50 \%$ dynamic load, has two unstable modes corresponding to the two positive eigenvalues at 3.6507 and 0.36118. The participation vectors corresponding to these two modes are given in Table 1. The participation vectors indicate that the states $\triangle E_{q}^{\prime}$ and $\triangle E_{m}^{\prime}$ make the most significant contributions in these unstable modes. These modes cannot be damped by a PSS. 
Table 1: Participation factors

\begin{tabular}{|c|c|c|c|c|c|c|c|}
\hline States & $\triangle E_{q}^{\prime}$ & $\triangle E_{m}^{\prime}$ & $\triangle S$ & $\triangle V_{o}$ & $\triangle \omega$ & $\triangle \delta$ & $\triangle \delta_{m}$ \\
\hline Part. factor for Mode 1 & 1.0 & 0.84676 & 0.31315 & 0.27738 & 0.14838 & 0.14838 & 0.02121 \\
\hline Part. factor for Mode 2 & 1.0 & 0.70158 & 0.08191 & 0.05818 & 0.04749 & 0.00773 & 0.00135 \\
\hline
\end{tabular}

A controller based on an approximate linearised model might fail to stabilise the system during recovery from large disturbances. To deal with this problem, we propose a robust stabilisation approach to the design of an excitation controller, with the objective of stabilising the response after relatively large disturbances. Using participation vector and eigenvector analyses, we show that such a system becomes unstable due to the voltage mode. In this design, the controlled variable and controller input-output are selected by the residue method which ensures that the voltage mode is stabilised by the designed controller. The block diagram of the proposed controller is given in Fig. 1 and, unlike for the PSS, the terminal voltage is used as the feedback signal. A coordinated PSS and voltage stability controller can be designed as suggested by the auxiliary input, $V_{s}$ in Fig. 1, but the focus of this work is the design of a robust voltage stability controller for large disturbances.

\section{Linearisation and uncertainty modelling}

A linearised model of a power system is usually obtained by expanding the right-handsides of the system differential equations, around an equilibrium point, in a Taylor series and retaining only the linear terms [32]. This linearisation technique limits the applicability of the linear model to small deviations from the equilibrium point. In general, the range of these small deviations is difficult to quantify. In this research, we use a robust linear control method to design stabilising control for large disturbances in power systems. To do so, we need to quantify the range of validity of linearised models. This can be done by the use of the Cauchy remainder formula for the Taylor series [32]. In this paper, in the design of the linear controller, the Cauchy remainder is incorporated as an uncertain term thus quantifying the deviations from the equilibrium point. Next, the linearisation process is described.

Let $\left(x_{0}, u_{0}\right)$ be an arbitrary point, using the mean-value theorem, the system (1)-(7) can 
be rewritten as follows [33]:

$$
\dot{x}=f\left(x_{0}, u_{0}\right)+L\left(x-x_{0}\right)+M\left(u-u_{0}\right)
$$

where

$$
L=\left[\left.\frac{\partial f_{1}}{\partial x}\right|_{\substack{x=x^{* 1} \\ u=u * 1}}, \ldots,\left.\frac{\partial f_{7}}{\partial x}\right|_{\substack{x=x^{* 7} \\ u=u * 7}}\right]^{T} \text {, and } M=\left[\left.\frac{\partial f_{1}}{\partial u}\right|_{\substack{x=x^{*} \\ u=u * 1}}, \ldots,\left.\frac{\partial f_{7}}{\partial u}\right|_{\substack{x=x^{* 7} \\ u=u * 7}}\right]^{T}
$$

Here $\left(x^{* p}, u^{* p}\right), p=1, \ldots, 7$, denote points lying on the line segment connecting points $(x, u)$ and $\left(x_{0}, u_{0}\right), f=\left[f_{1}, \ldots, f_{7}\right]^{T}$ denotes the vector function on the right-hand side of the differential equations (1)-(7). Equation (12) is an exact reformulation of the system (1)(7). The nonlinearity of the system $(1)-(7)$ is captured through the nonlinear dependencies $x^{* p}=\Phi_{1 p}\left(x, u, x_{0}, u_{0}\right)$ and $u^{* p}=\Phi_{2 p}\left(x, u, x_{0}, u_{0}\right), p=1, \ldots, 7$. It should be noted that the exact form of the functions $\Phi_{1 p}$ and $\Phi_{2 p}$ are not available, and due to this, instead of the exact expressions for $L$ and $M$ in system (12), their bounds are used in the control design.

Letting $\left(x_{0}, u_{0}\right)$ be the equilibrium point about which the trajectory is to be stabilised and defining $\Delta x \triangleq x-x_{0}$ and $\Delta u \triangleq u-u_{0}$, it is possible to rewrite (12) as follows:

$$
\begin{aligned}
& \Delta \dot{x}=\dot{x}-\dot{x_{0}}, \\
& =L\left(x-x_{0}\right)+M\left(u-u_{0}\right) \text {, } \\
& =\left[\left.\frac{\partial f_{1}}{\partial x}\right|_{\substack{x=x_{0} \\
u=u_{0}}}, \cdots,\left.\frac{\partial f_{7}}{\partial x}\right|_{\substack{x=x_{0} \\
u=u_{0}}}\right]^{T} \Delta x+\left(\left[\left.\frac{\partial f_{1}}{\partial x}\right|_{\substack{x=x^{* 1} \\
u=u^{* 1}}}, \cdots,\left.\frac{\partial f_{7}}{\partial x}\right|_{\substack{x=x^{* 7} \\
u=u^{* 7}}}\right]^{T}\right. \\
& \left.-\left[\left.\frac{\partial f_{1}}{\partial x}\right|_{\substack{x=x_{0} \\
u=u_{0}}}, \cdots,\left.\frac{\partial f_{7}}{\partial x}\right|_{\substack{x=x_{0} \\
u=u_{0}}}\right]^{T}\right) \Delta x+\left[\left.\frac{\partial f_{1}}{\partial u}\right|_{\substack{x=x^{* 1} \\
u=u^{* 7}}}, \cdots,\left.\frac{\partial f_{7}}{\partial u}\right|_{\substack{x=x^{* 7} \\
u=u^{* 7}}}\right]^{T} \Delta u, \\
& =A \triangle x+(L-A) \triangle x+B_{1} \triangle u \text {, }
\end{aligned}
$$

where $A=\left.\frac{\partial f}{\partial x}\right|_{\substack{x=x_{0} \\ u=u_{0}}}, B_{1}=\left.\frac{\partial f}{\partial u}\right|_{\substack{x=x_{0} \\ u=u_{0}}}$, and $\triangle x=\left[\triangle \delta, \triangle E_{q}^{\prime}, \triangle \omega, \triangle V_{t r}, \triangle \delta_{m}, \triangle E_{m}^{\prime}, \triangle S\right]^{T}$. Equation (13) is linear with respect to the control vector. Since $x^{* p}, p=1, \ldots, 7$ are not known, it is difficult to obtain the exact value of $(L-A)$, but it is possible to obtain a bound on $\|(L-A)\|$.

We rewrite system (13) in terms of the block diagram shown in Fig. 3. 


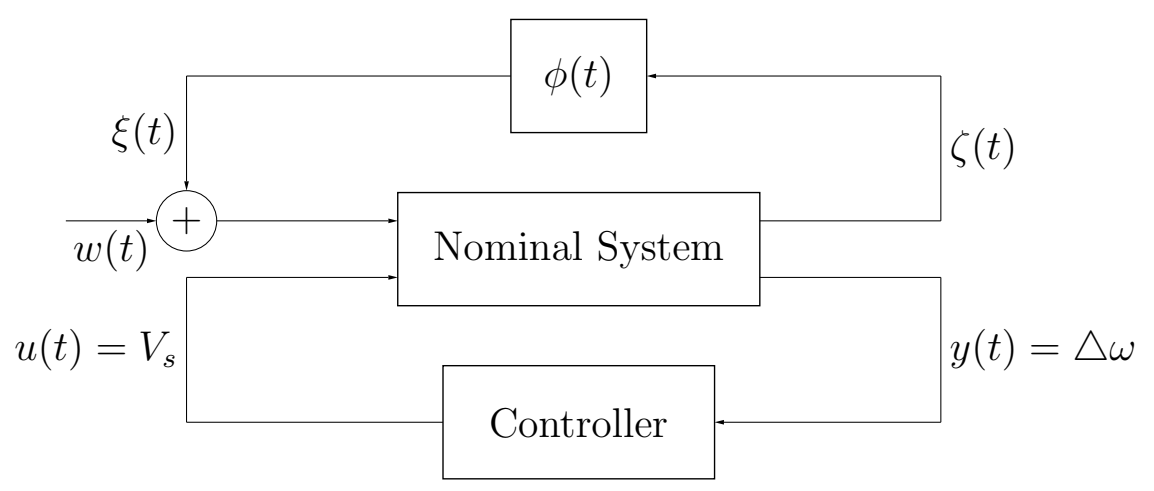

Figure 3: Control scheme

Let

$$
(L-A) \triangle x=B_{2} \xi, \quad \text { and } \quad \xi=\tilde{\phi}(t) \tilde{C}_{1} \triangle x,
$$

where $\xi(t)$ is known as the uncertainty input and $\tilde{\phi}(t)$ is a uncertain gain matrix. Equation (14) can be written as

$$
(L-A) \triangle x=B_{2} \tilde{\phi}(t) \tilde{C}_{1} \triangle x
$$

The system now can be rewritten as

$$
\triangle \dot{x}=A \triangle x+B_{1} \triangle u+B_{2} \xi .
$$

Matrices $B_{2}$ and $\tilde{C}_{1}$ are chosen as

$$
\begin{gathered}
B_{2}=\operatorname{diag}\left(0, \frac{X_{d}-X_{d}^{\prime}}{T_{d o}^{\prime}}, \frac{1}{2 H}, \frac{1}{T_{r}}, \frac{X-X^{\prime}}{T_{d o m}^{\prime}}, \frac{1}{2 H_{m}}, \frac{X-X^{\prime}}{T_{d o m}^{\prime}}\right), \\
\tilde{C}_{1}=\left[\begin{array}{lllllll}
1 & 0 & 0 & 0 & 0 & 0 & 0 \\
0 & 1 & 0 & 0 & 0 & 0 & 0 \\
0 & 0 & 0 & 0 & 1 & 0 & 0 \\
0 & 0 & 0 & 0 & 0 & 1 & 0
\end{array}\right],
\end{gathered}
$$

such that

$$
(L-A)=B_{2} \tilde{\phi}(t) \tilde{C}_{1} .
$$

The expressions for obtaining $\tilde{\phi}(t)$ are given in Appendix C.

Next we introduce a scaling parameter $\alpha$ and write

$$
\phi(t)=\frac{\tilde{\phi}(t)}{\sqrt{\alpha}}, \text { and } C_{1}=\sqrt{\alpha} \tilde{C}_{1} .
$$


Here $\alpha$ is a scaling factor which affects the magnitude of the uncertain output $\zeta$ defined as $\zeta=C_{1} \triangle x$

Finally the value of $\alpha$ is chosen such that the uncertainty, $\phi(t)$, shown in Fig. 3 satisfies,

$$
\|\phi(t)\|^{2} \leq 1
$$

From this we have

$$
\|\xi\|^{2} \leq \alpha\left\|\tilde{C}_{1} \triangle x\right\|^{2}
$$

and we recover the following norm bound constraints [29],

$$
\|\xi\|^{2} \leq\|\zeta\|^{2}
$$

To facilitate control design, the power system model is summarised as

$$
\begin{aligned}
\triangle \dot{x}(t) & =A \triangle x(t)+B_{1} \triangle u(t)+B_{2} \xi(t)+B_{2} w(t) \\
y(t) & =C_{2} \triangle x(t)+D_{2} \xi(t)+D_{2} w(t) \\
\zeta(t) & =C_{1} \triangle x(t)
\end{aligned}
$$

where $\zeta=\left[\triangle \delta^{*}, \triangle E_{q}^{*^{\prime}}, \triangle \delta_{m}^{*}, \triangle E_{m}^{*^{\prime}}\right]^{T}$, and it is known as the uncertainty output, $y(t)$ is the measured output, and $w(t)$ is a unity covariance Gaussian white noise process corresponding to the nominal disturbance. The output matrix $C_{2}$ is defined as

$$
C_{2}=\left[\begin{array}{lllllll}
0 & 0 & 0 & 1 & 0 & 0 & 0
\end{array}\right]
$$

Equations (23)-(25) provide a new representation of the power system model with a linear part, and another part with higher-order terms. The new formulation presented in this section is used with the minimax LQG control theory to design a voltage controller for the nonlinear power system.

\section{Minimax LQG control}

In this paper, we use a simplified version of the main results of [34] concerning the minimax LQG control problem. A complete description of this method can be found in [29]. In this paper, the minimax LQG method is applied to uncertain systems of the form shown in Fig. 3. 
Associated with the uncertain system (23)-(25), we consider a cost functional $J$ of the form

$$
J=\lim _{T \rightarrow \infty} \frac{1}{2 T} E \int_{0}^{T}\left(\triangle x(t)^{T} R \triangle x(t)+\triangle u(t)^{T} G \triangle u(t)\right) d t,
$$

where $R \geq 0$ and $G>0, R \in R^{n \times n}, G \in R^{m \times m}$ and $E$ is the expectation operator.

The quadratic cost (27) is particularly suited to the design of an excitation controller for the power system. Every generator has an overexcitation limiter which limits the field voltage based on time integral of the voltage. This means that one can apply large voltages as long as they are for short duration. Thus the quadratic cost optimisation is much more suitable in this situation as compared to $H_{\infty}$-norm based designs.

The minimax optimal control finds the controller which minimises $J$ over all admissible uncertainties. The cost function $J$ satisfies the following relationship [29]:

$$
\sup _{\|\xi\|^{2} \leq\|\zeta\|^{2}} J\left(u^{*}\right) \leq \inf _{\tau} V_{\tau}
$$

where $V_{\tau}$ is given by

$$
V_{\tau}=\frac{1}{2} \operatorname{tr}\left[Y_{\infty} R_{\tau}+\left(Y_{\infty} C_{2}^{T}+B_{2} D_{2}^{T}\right)\left(D_{2} D_{2}^{T}\right)^{-1}\left(C_{2} Y_{\infty}+D_{2} B_{2}^{T}\right) X_{\infty}\left(I-\frac{1}{\tau} Y_{\infty} X_{\infty}\right)^{-1}\right]
$$

$\tau$ is a free parameter and the matrices $X_{\infty}$ and $Y_{\infty}$ are the solution to the following pair of parameter dependent algebraic Riccati equations [29]:

$$
\begin{aligned}
& \left(A-B_{2} D_{2}^{T}\left(D_{2} D_{2}^{T}\right)^{-1} C_{2}\right) Y_{\infty}+Y_{\infty}\left(A-B_{2} D_{2}^{T}\left(D_{2} D_{2}^{T}\right)^{-1} C_{2}\right)^{T}- \\
& Y_{\infty}\left(C_{2}^{T}\left(D_{2} D_{2}^{T}\right)^{-1} C_{2}-\frac{1}{\tau} R_{\tau}\right) Y_{\infty}+B_{2}\left(I-D_{2}^{T}\left(D_{2} D_{2}^{T}\right)^{-1} D_{2}\right) B_{2}^{T}=0
\end{aligned}
$$

and

$$
\begin{aligned}
& X_{\infty}\left(A-B_{1} G_{\tau}^{-1} \gamma_{\tau}^{T}+\left(A-B_{1} G_{\tau}^{-1} \gamma_{\tau}^{T}\right) X_{\infty}+\right. \\
& \left(R_{\tau}-\gamma_{\tau} G_{\tau}^{-1} \gamma_{\tau}^{T}\right)-X_{\infty}\left(B_{1} G_{\tau}^{-1} B_{1}^{T}-\frac{1}{\tau} B_{2} B_{2}^{T}\right) X_{\infty}=0
\end{aligned}
$$

The solutions are required to satisfy the following conditions: $Y_{\infty}>0, X_{\infty}>0$, the spectral radius of the matrix $X_{\infty} Y_{\infty}$ is $\rho\left(X_{\infty} Y_{\infty}\right)<\tau, R_{\tau}-\gamma_{\tau}^{T} G_{\tau}^{-1} \gamma_{\tau} \geq 0, R_{\tau}=R+\tau C_{1}^{T} C_{1}, G_{\tau}=$ $G+\tau D_{1}^{T} D_{1}$, and $\gamma_{\tau}=\tau C_{1}^{T} D_{1}$

To obtain the minimax LQG controller, the parameter $\tau>0$ is chosen to minimise $V_{\tau}$. A line search is carried out to find the value of $\tau>0$ which attains the minimum value of 
the cost function $V_{\tau}$. This line search involves solving the Riccati equations (30) and (31) for different values of $\tau$ and finding the value which gives the smallest $V_{\tau}$.

The minimax LQG optimal controller is given by the equations:

$$
\begin{aligned}
& \dot{\hat{x}}_{c}=\left(A-B_{1} G_{\tau}^{-1} \gamma_{\tau}^{T}\right) \hat{x}_{c}-\left(\left(B_{1} G_{\tau}^{-1} B_{1}^{T}-\frac{1}{\tau} B_{2} B_{2}^{T}\right) X_{\infty}\right) \hat{x}_{c}+\left(I-\frac{1}{\tau} Y_{\infty} X_{\infty}\right)^{-1} \\
& \left(Y_{\infty} C_{2}^{T}+B_{2} D_{2}^{T}\right) \times\left(D_{2} D_{2}^{T}\right)^{-1}\left(y-\left(C_{2}+\frac{1}{\tau} D_{2} B_{2}^{T} X_{\infty}\right) \hat{x_{c}}\right) \\
& u=-G_{\tau}^{-1}\left(B_{1}^{T} X_{\infty}+\gamma_{\tau}^{T}\right) \hat{x}_{c} .
\end{aligned}
$$

In the next section we design a minimax LQG controller for the test power system and evaluate its performance.

\section{Controller design and performance evaluation}

Prior to the controller design, we carry out several large disturbance simulations to get an idea of the region of interest. The maximum value of $\phi(t)$ is obtained over this region and not globally. If the maximum value of $\phi(t)$ is evaluated over the entire uncertainty region, the calculation burden will be very high and it will lead to a conservative controller. This method to evaluate the maximum value of $\phi(t)$ is different from the global maximum method used in [28]. The controller is then designed as follows:

Step 1 From the simulations of the faulted system, obtain the range of variations of all state variables and form a volume, $\Omega$, with corner points given by $\left(x_{f_{p}}-x_{0_{p}}\right), p=1, \ldots, 7$, where $x_{f_{p}}$ is the largest variation of the $p^{t h}$ state variable about its equilibrium value, $x_{0_{p}}$. Formally $x \in \Omega$ if $\left|x-x_{0_{p}}\right| \leq\left|x_{f_{p}}-x_{0_{p}}\right|$.

\section{Step 2 Obtain}

$$
\alpha^{*}=\max _{x^{* p} \in \Omega}\left\{\alpha:\|\phi(t)\|^{2}<1\right\} .
$$

The process to obtain $\alpha^{*}$ involves obtaining the maximum value of $\|\tilde{\phi}(t)\|$ over the volume $\Omega$.

Step 3 Check if there exists a feasible controller with $\alpha=\alpha^{*}$, i.e., if there is a scalar $\tau$ such that there is a feasible solution to the coupled Riccati equations (30) and (31). 
Step 4 If we obtain a feasible controller in the above step, either enlarge the volume, $\Omega$, i.e., increase the operating region of the controller or if we have arrived at the largest possible volume, perform an optimal search over the scalar parameter, $\tau$, to get the infimum of $V_{\tau}$. If there is no feasible solution with the chosen $\alpha=\alpha^{*}$, reduce the volume, $\Omega$, and go to Step 2.

The term $x(t)^{\prime} R x(t)$, in the cost function (27), corresponds to the norm-squared value of the nominal system output and $u^{\prime} G u$ is treated as a design parameter affecting controller gain. Although a certain amount of trial-and-error is needed, it is determined that the value of $G=10^{-4}$ and the matrix $R=\operatorname{diag}(1,3,1,1,1,2,1)$ in the cost function (27) ensure the satisfactory performance of the controller. It can be seen that the state variable representing the transient emf is given higher weight compared with those of other state variables since we want a quick controller response to compensate for the reactive power mismatch. Furthermore, the theory requires that $D_{2} D_{2}^{\prime}>0[34]$, so we choose $D_{2}=10^{-6}[1,1,1,1]$.

The process described above enables the selection of the largest range for which a feasible controller is obtained. For the given power system model, we obtain the value of $\alpha^{*}=0.64$, for the range of $\left(x^{* p}-x^{p}\right)$ given in Table 2 and the optimum value of the parameter $\tau$ is 3.42 .

Table 2: Operating region

\begin{tabular}{|c|c|c|c|c|c|c|c|}
\hline$\left|E_{m}^{*^{\prime}}-E_{m o}\right|$ & $\left|E_{q}^{*^{\prime}}-E_{q o}\right|$ & $\left|V_{t r}^{*}-V_{t r o}\right|$ & $\left|\omega^{*}-\omega_{0}\right|$ & $\left|\delta^{*}-\delta_{0}\right|$ & $\left|\delta_{m}^{*}-\delta_{m o}\right|$ & $\left|S^{*}-S_{o}\right|$ & $\alpha$ \\
\hline $0.25 \mathrm{pu}$ & $0.25 \mathrm{pu}$ & $0.275 \mathrm{pu}$ & $0.345 \mathrm{pu}$ & $76.18^{0}$ & $82.38^{0}$ & $0.225 \mathrm{pu}$ & 0.64 \\
\hline
\end{tabular}

The eigenvalues of the closed loop system are shown in Table 3. The dominant mode for the closed loop system is $-1.189 \pm \jmath 1.8794$ and the damping ratio is 0.12971 . From the eigenvalues it is clear that the closed loop system is well-damped. The performance of the designed robust excitation controller is tested by simulating response to three contingencies on the test system. The contingencies are: (I) the outage of one transmission line, (II) threephase short circuit at one of the transmission lines between buses 3 and 4 , and (III) sudden change in load power. The simulation sampling time is $2.5 \mathrm{~ms}$ for all cases.

Table 3: Closed loop eigenvalues

\begin{tabular}{|l|l|l|l|}
\hline$-2.946 \pm i 6.14$ & $-4.846 \pm i 5.46$ & $-1.189 \pm i 3.16$ & -27.470 \\
\hline
\end{tabular}




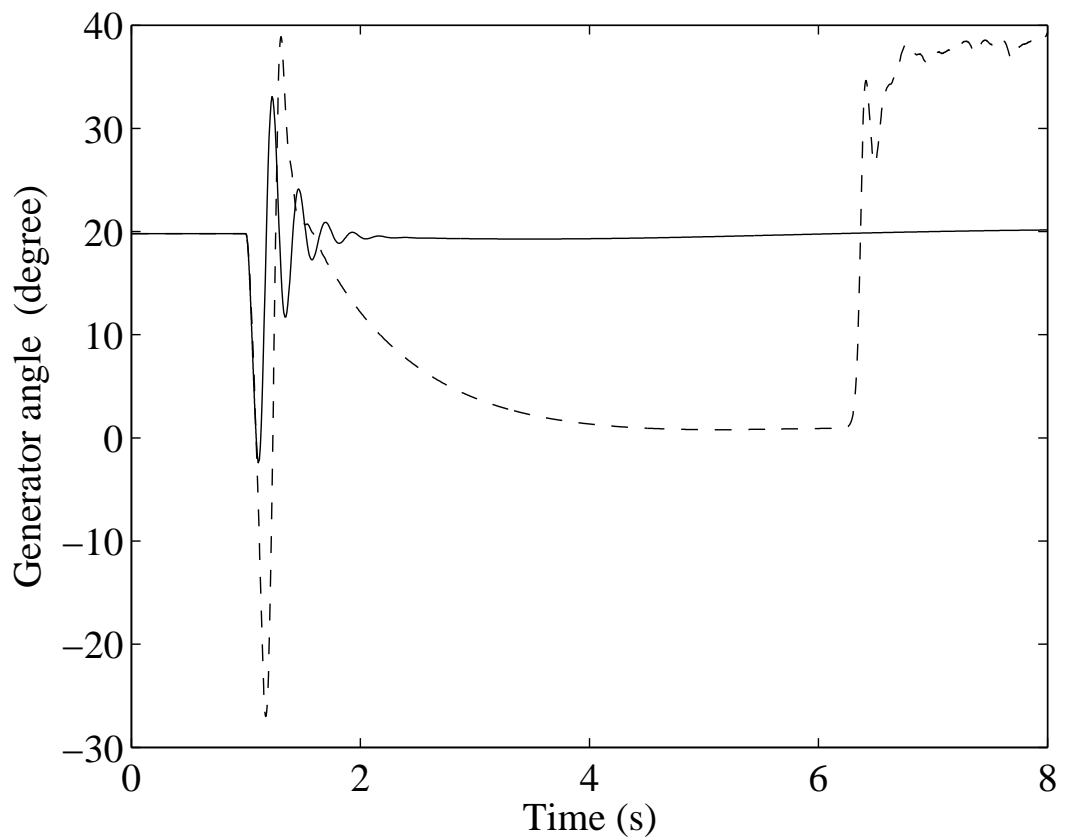

Figure 4: Generator angle - outage of one line (Solid line designed controller and dash line IEEE ST1A exciter)

\subsection{Contingency I: Outage of one transmission line}

A transmission line outage increases line impedance and weakens interconnections. Due to the increase in line reactance, extra reactive power is needed in order to maintain the voltage at the load bus. The AVR responds to this condition by increasing the generator field voltage which has a beneficial effect on voltage stability. The effectiveness of this type of control depends on the ability of the excitation system to quickly increase the field voltage to meet the required reactive power.

Here, a transmission line outage simulation is performed by opening the line at 1 s and reclosing it after five cycles. Figs. 4 and 5 show the generator angle and terminal voltage response, respectively, with the designed excitation controller and an IEEE ST1A exciter. The simulation is repeated for the same contingency with a $25 \%$ dynamic load for which the terminal voltage of the generator is shown in Fig. 6. From the responses, it can be concluded that the designed controller can stabilise the voltage as well as the angle of the generator within $2 \mathrm{~s}$ of this large disturbance. Although the excitation controller is designed with the load being $50 \%$ dynamic and $50 \%$ static, it also performs well if the composition of the total load includes less than a $50 \%$ dynamic load. 


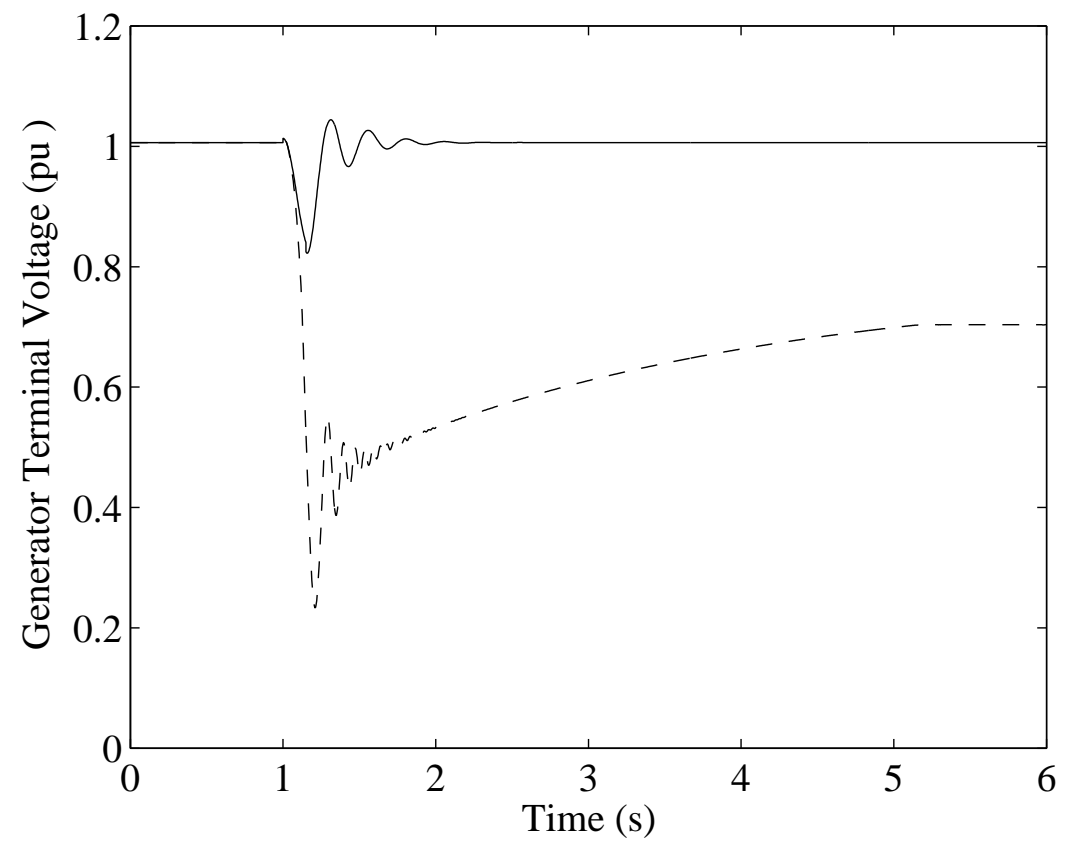

Figure 5: Generator terminal voltage (50\% dynamic load) — outage of one line (Solid line designed controller and dash line IEEE ST1A exciter)

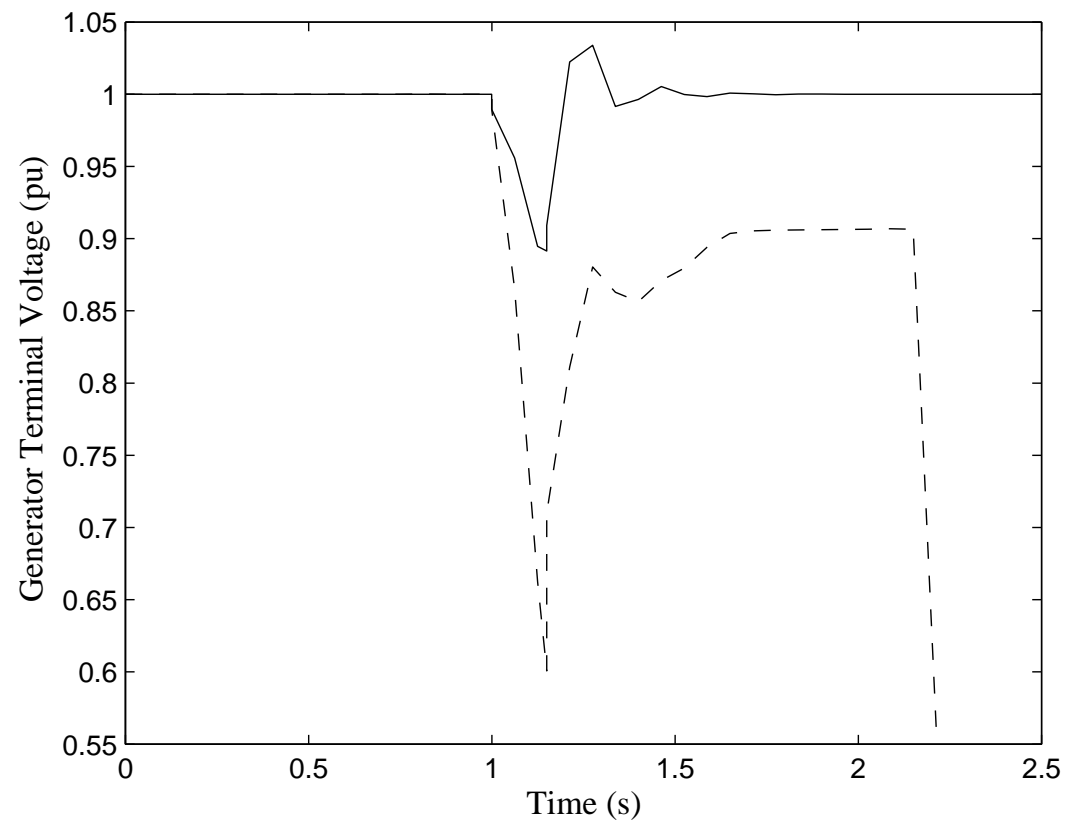

Figure 6: Generator terminal voltage (25\% dynamic load) - outage of one line (Solid line designed controller and dash line IEEE ST1A exciter) 


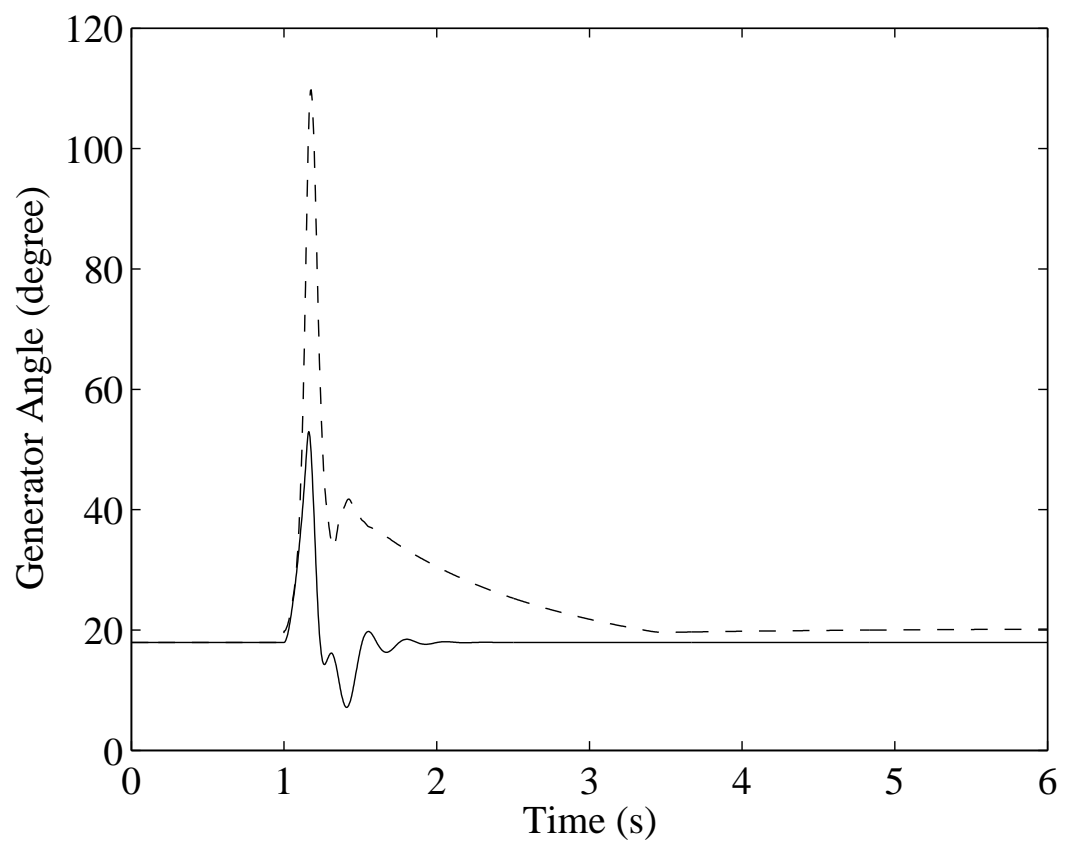

Figure 7: Generator angle-three-phase fault (Solid line designed controller and dash line IEEE ST1A exciter)

\subsection{Contingency II: Three-phase short circuit}

One of the most severe disturbances, perhaps leading to voltage collapse, is a three-phase fault on one of the key transmission circuits. From the power system viewpoint, the excitation system should contribute to the effective control of voltage and the enhancement of system stability. The excitation system should be capable of responding rapidly to a disturbance by modulating the generator field so as to enhance voltage stability.

Here, a simulation is performed for a three-phase fault on one of the transmission lines. The fault is cleared by auto-reclosing the circuit breaker after 0.15 s. Figs. 7 and 8 show the angle and terminal voltage responses of the local generator, respectively, from which it can be seen that the proposed controller stabilises voltage within five cycles of a fault occurrence and damps out the power angle oscillations.

\subsection{Contingency III: Sudden change in load}

The load characteristics and dynamics indicate the dependency between the load and the voltage, and the close coupling of the load dynamics with the voltage stability phenomenon. The stable operation of a power system depends on its ability to continuously match the electrical output of generating units to the electrical load on the system. As loads vary, the 


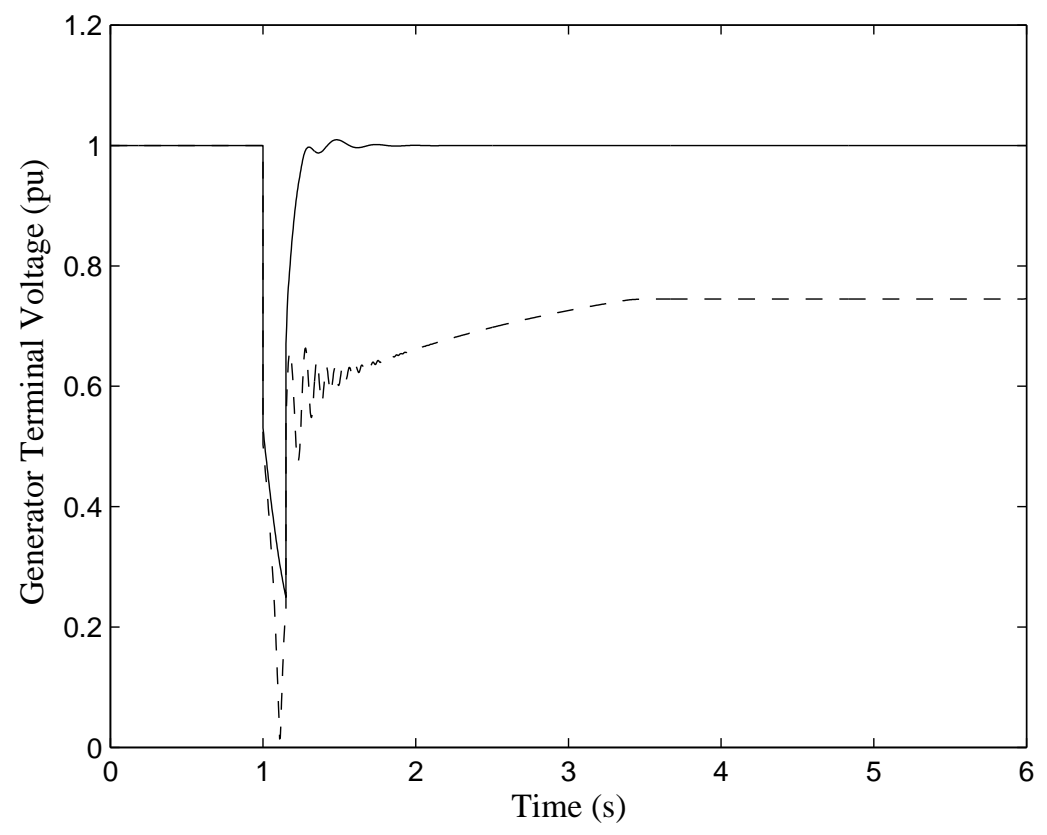

Figure 8: Generator terminal voltage - three phase-fault (Solid line designed controller and dash line IEEE ST1A exciter)

reactive power requirements of the transmission system also vary. In practice, a power system experiences continuous changes in load demand which can cause voltage instability unless an appropriate control action is taken.

The performance of the designed controller is also evaluated for a less severe fault, such as a sudden change in load. At $1 \mathrm{~s}$, the load is increased by $5 \%$ and the transient responses of the local generator and the load voltage due to this change are shown in Figs. 9 and 10, respectively, in which it is clear that both the controllers can stabilise the system against a less severe disturbance but that the proposed controller performs better in terms of settling time, damping, and oscillations.

The simulation is repeated for a comparatively severe disturbance, i.e., a $25 \%$ change in load. In this case, although the designed controller requires more settling time to stabilise the system, it still provides good performance. On the other hand, the IEEE ST1A cannot stabilise the system so the voltage collapses and the generator goes out of synchronism.

From the above analyses, it is clear that the proposed controller ensures a wider stable operating region and stabilises the system against large disturbances with improved settling 


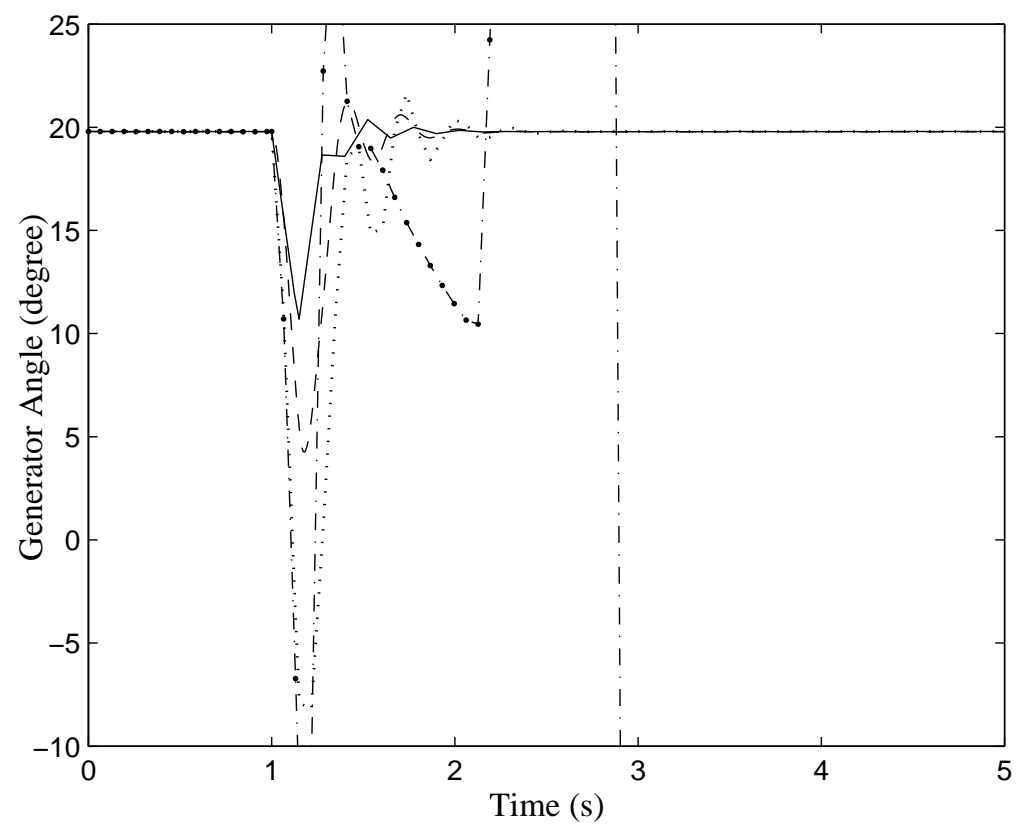

Figure 9: Generator angle- change in load power (For 5\% and 25\% change in load, solid and dot line designed controller, and the dash and dash-dot line IEEE ST1A exciter)

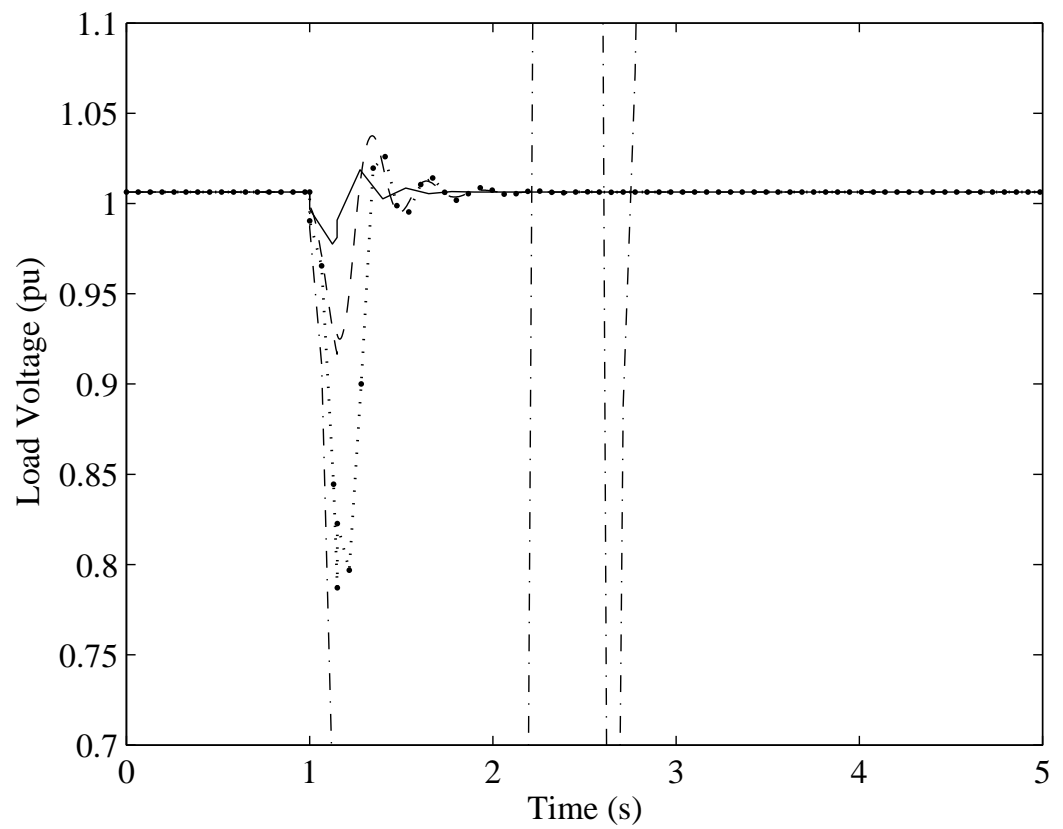

Figure 10: Load voltage- change in load power ( For $5 \%$ and 25\% change in load, solid and dot line designed controller, and the dash and dash-dot line IEEE ST1A exciter) 
time, damped oscillations, and a smaller steady state error. The conventional controller can stabilise the power system against less severe disturbances but fails to do so when large disturbances occur.

\section{Conclusions and future work}

In this paper an algorithm to design a robust output feedback excitation controller is proposed. Detailed modelling of each component and a suitable control strategy for large disturbances in power systems is presented. The excitation control scheme is based on the reformulation of the nonlinear dynamics of the generators and motors using the mean-value

theorem. With this representation, it becomes easier to explicitly account for the effect of nonlinearities in the system dynamics, which enables us to more accurately represent the system and also provides guaranteed performance and stability characteristics over a prespecified region around the equilibrium point. The effectiveness of the proposed control system is verified by applying several large disturbances. The performance of the proposed robust excitation controller is compared with an IEEE ST1A exciter and simulation results confirm the efficacy of the proposed control over the conventional excitation control. The next step of this research is to apply our proposed control technique to a real power system.

Future studies will deal with extending the proposed method to handle multi-machine power systems. The nonlinear effects for different conventional generators, wind generators, induction motors and FACTS devices, will be accounted for in a way similar to this paper, using the mean-value theorem. This will require an extension of our results to the decentralised control framework for generator excitation systems and FACTS devices. 


\section{Appendix A}

Let

$E_{m}^{\prime}=\sqrt{e_{d m}^{\prime 2}+e_{q m}^{\prime 2}}, \delta_{m}=\tan ^{-1} \frac{-e_{d m}^{\prime}}{e_{q m}^{\prime}},\left(I_{d m}+j I_{q m}\right)=-\left(i_{d m}+j i_{q m}\right) e^{-j \delta_{m}},\left(V_{d}+j V_{q}\right)=$ $\left.v_{d}+j v_{q}\right) e^{-j \delta_{m}}, T_{m}=-T_{L}, T_{e}=e_{q m}^{\prime} i_{q m}+e_{q m}^{\prime} i_{q m}, i_{d m}=-I_{d m} \cos \delta+I_{q m} \sin \delta$, and $i_{q m}=$ $-I_{q m} \cos \delta-I_{d m} \sin \delta$.

Taking derivative of $E_{m}^{\prime}$, we get

$$
\begin{aligned}
\dot{E}_{m}^{\prime} & =\frac{1}{2 \sqrt{e_{d m}^{\prime 2}+e_{q m}^{\prime 2}}}\left(2 e_{d m}^{\prime} \dot{e}_{d m}^{\prime}+2 e_{q m}^{\prime} \dot{e}_{q m}^{\prime}\right) \\
& =\frac{1}{T_{d o m}^{\prime} \sqrt{e_{d m}^{\prime 2}+e_{q m}^{\prime 2}}}\left[-e_{d m}^{\prime 2}-e_{q m}^{\prime 2}-\left(X-X^{\prime}\right)\left(e_{d m}^{\prime} i_{q m}-e_{q m}^{\prime} i_{d m}\right]\right. \\
& =\frac{1}{T_{d o m}^{\prime}}\left[-E_{m}^{\prime}-\left(X-X^{\prime}\right) \frac{e_{d m}^{\prime} i_{q m}-e_{q m}^{\prime} i_{d m}}{\sqrt{e_{d m}^{\prime 2}+e_{q m}^{\prime 2}}}\right] \\
& =\frac{1}{T_{d o m}^{\prime}}\left[-E_{m}^{\prime}-\left(X-X^{\prime}\right) I_{d m}^{\prime}\right]
\end{aligned}
$$

$$
\begin{aligned}
T_{e} & =e_{q m}^{\prime}\left(-I_{d m} \cos \delta+I_{q m} \sin \delta\right)+e_{q m}^{\prime}\left(-I_{q m} \cos \delta-I_{d m} \sin \delta\right) \\
& =-e_{q m}^{\prime} I_{d m} \frac{e_{d m}^{\prime}}{\sqrt{e_{d m}^{\prime 2}+e_{q m}^{\prime 2}}}+e_{q m}^{\prime} I_{q m} \frac{e_{q m}^{\prime}}{\sqrt{e_{d m}^{\prime 2}+e_{q m}^{\prime 2}}}=-E_{m}^{\prime} I_{d m} .
\end{aligned}
$$

Differentiating $\delta_{m}$, we get

$$
\begin{aligned}
\dot{\delta}_{m}= & \frac{1}{1+\left(\frac{e_{d m}^{\prime}}{e_{q m}^{\prime}}\right)^{2}} \times \frac{e_{q m}^{\prime} \dot{e}_{d m}^{\prime}-e_{d m}^{\prime} \dot{e}_{q m}^{\prime}}{e_{q m}^{\prime 2}} \\
= & \frac{e_{q m}^{2}}{e_{d m}^{\prime 2}+e_{q m}^{\prime 2}} \times 1 / e_{q m}^{\prime 2}\left[-e_{q m}^{\prime} e_{d m}^{\prime}+e_{q m}^{\prime} e_{d m}^{\prime}\right. \\
& \left.-\left(X-X^{\prime}\right)\left(e_{q m}^{\prime} i_{q m}+e_{d m}^{\prime} i_{d m}\right)+T_{d o m}^{\prime} \omega_{s}(S-1)\left(e_{d m}^{\prime 2}+e_{q m}^{\prime 2}\right)\right] \\
= & S \omega_{s}-\omega_{s}-\frac{X-X^{\prime}}{T_{d o m}^{\prime} E_{m}^{\prime}} I_{q m} .
\end{aligned}
$$




\section{Appendix B}

Table 4: System parameters (in pu)

\begin{tabular}{|c|c|c|c|}
\hline Machine & Motor & System & AVR \\
\hline$X_{d}=2.1$ & $X_{s}=0.1$ & $X_{\infty}=0.01$ & $K_{a}=100$ \\
$X_{q}=2.1$ & $X_{r}=0.18$ & $X_{34}=0.0554$ & $T_{a}=0.1 s$ \\
$X_{d}^{\prime}=0.4$ & $X_{m}=3.2$ & $X_{14}=0.016$ & $E_{f d}^{\min }=0$ \\
$T_{d o}^{\prime}=8 \mathrm{~s}$ & $R_{r}=0.018$ & $X_{24}=0.004$ & $E_{f d}^{\max }=5$ \\
$\omega_{s}=377$ & $H_{m}=0.5$ & & \\
$H=3.5$ & & & \\
$D=4$ & & & \\
\hline
\end{tabular}

The synchronous machine parameters are on a 500 MVA base, the induction motor parameters are on a 800-MVA base, the exciter on a 100 MVA base and the system reactances are on a 100-MVA base.

\section{Appendix C}

We define $\tilde{\phi}=\left[\begin{array}{lll}\tilde{\phi}_{1} & \cdots & \tilde{\phi}_{7}\end{array}\right]^{T}$. The expressions for $\left[\begin{array}{lll}\tilde{\phi}_{1} & \cdots & \tilde{\phi}_{7}\end{array}\right]^{T}$ are given below:

$$
\begin{aligned}
& \tilde{\phi}_{1}=\left[\begin{array}{llll}
0 & 0 & 0 & 0
\end{array}\right] . \\
& \tilde{\phi}_{2}=\left[\begin{array}{llll}
x_{11} & x_{12} & x_{13} & x_{14}
\end{array}\right]\left[\begin{array}{llll}
y_{11} & 0 & 0 & 0 \\
y_{21} & 0 & 0 & 0 \\
y_{31} & 0 & y_{33} & y_{34} \\
y_{41} & 0 & y_{43} & y_{44}
\end{array}\right],
\end{aligned}
$$

where

$$
\begin{array}{lll}
x_{11}=\cos \delta^{*}-\cos \delta_{0}, & y_{11}=-V_{\infty} G_{13}, & y_{33}=-y_{31}, \\
x_{12}=\sin \delta^{*}-\sin \delta_{0}, & y_{21}=-V_{\infty} B_{13}, & y_{43}=-y_{41}, \\
x_{13}=\cos \left(\delta_{m}^{*}-\delta^{*}\right)-\cos \left(\delta_{m 0}-\delta_{10}\right), & y_{31}=-\left(E_{m}^{*^{\prime}}-E_{m 0}^{\prime}\right) G_{12}, & y_{34}=B_{12}, \\
x_{14}=-\sin \left(\delta_{m}^{*}-\delta^{*}\right)+\sin \left(\delta_{m 0}-\delta_{10}\right), & y_{41}=-\left(E_{m}^{*^{\prime}}-E_{m 0}^{\prime}\right) B_{12}, & y_{44}=G_{12} .
\end{array}
$$




$$
\tilde{\phi}_{3}=\left[\begin{array}{llll}
a_{11} & a_{12} & a_{13} & a_{14}
\end{array}\right]\left[\begin{array}{cccc}
b_{11} & b_{12} & 0 & 0 \\
b_{21} & b_{22} & 0 & 0 \\
b_{31} & b_{32} & b_{33} & b_{34} \\
b_{41} & b_{42} & b_{43} & b_{44}
\end{array}\right]+\frac{1}{2 H}\left[\begin{array}{llll}
0 & -2 G_{11}\left(E_{q}^{*^{\prime}}-E_{q 0}^{\prime}\right) & 0 & 0
\end{array}\right],
$$

where

$$
\begin{aligned}
& a_{11}=-\sin \delta^{*}+\sin \delta_{0}, \\
& b_{11}=-V_{\infty}\left(E_{q}^{*^{\prime}}-E_{q 0}^{\prime}\right) G_{13}, \quad b_{33}=-b_{31}, \\
& a_{12}=\cos \delta^{*}-\cos \delta_{0}, \\
& b_{12}=V_{\infty} B_{13} \text {, } \\
& b_{34}=-b_{32} \text {, } \\
& a_{13}=\sin \left(\delta_{m}^{*}-\delta^{*}\right)-\sin \left(\delta_{m 0}-\delta_{0}\right) \\
& b_{21}=-V_{\infty}\left(E_{q}^{*^{\prime}}-E_{q 0}^{\prime}\right) B_{13}, \quad b_{43}=-b_{41}, \\
& \left.a_{14}=\cos \left(\delta_{m}^{*}-\delta^{*}\right)-\cos \left(\delta_{m 0}-\delta_{0}\right)\right) \\
& b_{22}=-V_{\infty} G_{13} \text {, } \\
& b_{44}=-b_{42} \text {, } \\
& b_{31}=-\left(E_{m}^{*^{\prime}} E_{q}^{*^{\prime}}-E_{m 0}^{\prime} E_{q 0}^{\prime}\right) G_{12} \\
& b_{32}=\left(E_{m}^{*^{\prime}}-E_{m 0}^{\prime}\right) B_{12}, \\
& b_{42}=-\left(E_{m}^{*^{\prime}}-E_{m 0}^{\prime}\right) G_{12}, \\
& b_{41}=-\left(E_{m}^{*^{\prime}} E_{q}^{*^{\prime}}-E_{m 0}^{\prime} E_{q 0}^{\prime}\right) B_{12} \text {. } \\
& \tilde{\phi}_{4}=\left[\begin{array}{llll}
x_{11} & x_{12} & x_{13} & x_{14}
\end{array}\right] \beta\left[\begin{array}{cccc}
b_{11} & b_{22} & 0 & 0 \\
b_{21} & b_{22} & 0 & 0 \\
b_{31} & b_{32} & b_{33} & b_{34} \\
b_{41} & b_{42} & b_{43} & b_{44}
\end{array}\right]+\frac{1}{2 H}\left[\begin{array}{l}
x_{11} \\
x_{12} \\
x_{13} \\
x_{14}
\end{array}\right]^{T} \kappa\left[\begin{array}{llll}
p_{11} & p_{12} & 0 & 0 \\
p_{21} & p_{22} & 0 & 0 \\
p_{31} & p_{32} & p_{33} & p_{34} \\
p_{41} & p_{42} & p_{43} & p_{44}
\end{array}\right] \\
& +\left[\begin{array}{llll}
0 & q_{2}\left(\frac{E_{q}^{*^{\prime}}}{V_{t}^{*}}-\frac{E_{q 0}^{\prime}}{V_{t 0}}\right) & 0 & 0
\end{array}\right],
\end{aligned}
$$

where

$$
\begin{array}{ll}
p_{11}=-V_{\infty}\left(E_{q}^{*^{\prime}}-E_{q 0}^{\prime}\right) G_{11} G_{\infty}+B_{11} B_{13}, & p_{12}=V_{\infty}\left(G_{11} B_{13}-B_{11} G_{13}\right), \\
p_{31}=-\left(E_{q}^{*^{\prime}} E_{m}^{*^{\prime}}-E_{q 0} E_{m 0}^{\prime}\right)\left(G_{11} G_{12}+B_{11} B_{12}\right), & \beta=X_{d}^{\prime}\left(\frac{1}{V_{t}^{*}}-\frac{1}{V_{t 0}}\right), \\
p_{21}=-V_{\infty}\left(E_{q}^{*^{\prime}}-E_{q 0}^{\prime}\right)\left(G_{11} B_{13}-B_{11} G_{13}\right), & \left.p_{22}=-V_{\infty} G_{11} G_{\infty}+B_{11} B_{13}\right), \\
p_{41}=-\left(E_{q}^{*^{\prime}} E_{m}^{*^{\prime}}-E_{q 0} E_{m 0}^{\prime}\right)\left(G_{11} B_{12}-B_{11} G_{12}\right), & p_{32}=\left(E_{m}^{*^{\prime}}-E_{m 0}^{\prime}\right)\left(G_{11} B_{12}-B_{11} G_{12}\right), \\
p_{42}=-\left(E_{m}^{*^{\prime}}-E_{m 0}^{\prime}\right)\left(G_{11} G_{12}+B_{11} B_{12}\right), & q_{2}=1+2 X_{d}^{\prime} B_{11}+X_{d}^{\prime 2}\left(G_{11}^{2}+B_{11}^{2}\right), \\
\kappa=X_{d}^{\prime} \beta, & p_{33}=-p_{31}, \\
p_{44}=-p_{42}, & p_{43}=-p_{41}, \\
p_{44}=-p_{42} . &
\end{array}
$$




$$
\tilde{\phi}_{5}=\left[\begin{array}{llll}
k_{11} & k_{12} & k_{13} & k_{14}
\end{array}\right]\left[\begin{array}{cccc}
0 & 0 & m_{13} & 0 \\
0 & 0 & m_{23} & 0 \\
m_{31} & m_{32} & m_{33} & 0 \\
m_{41} & m_{42} & m_{43} & 0
\end{array}\right],
$$

where

$$
\begin{array}{rlrl}
k_{11}=\cos \delta_{m}^{*}-\cos \delta_{m 0}, & m_{13}=-V_{\infty} G_{31}, & m_{33}=-m_{31}, \\
k_{12}=\sin \delta_{m}^{*}-\sin \delta_{m 0}, & m_{23}=-V_{\infty} B_{31}, & m_{41}=\left(E_{q}^{*^{\prime}}-E_{q 0}^{\prime}\right) B_{12}, \\
k_{13}=\cos \left(\delta^{*}-\delta_{m}^{*}\right)-\cos \left(\delta_{0}-\delta_{m 0}\right), & m_{31}=\left(E_{q}^{*^{\prime}}-E_{q 0}^{\prime}\right) G_{12}, & m_{42}=-G_{12}, \\
k_{14}=-\sin \left(\delta^{*}-\delta_{m}^{*}\right)+\sin \left(\delta_{0}-\delta_{m 0}\right), & m_{32}=B_{12}, & m_{43}=-m_{41} . \\
\tilde{\phi}_{6}=\left[\begin{array}{llll}
c_{11} & c_{12} & c_{13} & c_{14}
\end{array}\right] \gamma\left[\begin{array}{llll}
0 & 0 & d_{13} & d_{14} \\
0 & 0 & d_{23} & d_{24} \\
d_{31} & d_{32} & d_{33} & d_{34} \\
d_{41} & d_{42} & d_{43} & d_{44}
\end{array}\right]
\end{array}
$$

where

$$
\begin{array}{llll}
c_{11}=-\sin \delta_{m}^{*}+\sin \delta_{m 0}, & d_{13}=-V_{\infty}\left(E_{m}^{*^{\prime}}-E_{m 0}^{\prime}\right) G_{31}, & d_{34}=-d_{32}, \\
c_{12}=\cos \delta_{m}^{*}-\cos \delta_{m 0}, & d_{14}=-V_{\infty} B_{31}, & d_{33}=-d_{31}, \\
c_{13}=\sin \left(\delta^{*}-\delta_{m}^{*}\right)-\sin \left(\delta_{0}-\delta_{m 0}\right), & d_{23}=-V_{\infty}\left(E_{m}^{*^{\prime}}-E_{m 0}^{\prime}\right) B_{31}, & d_{43}=-d_{41}, \\
c_{14}=\cos \left(\delta^{*}-\delta_{m}^{*}\right)-\cos \left(\delta_{0}-\delta_{m 0}\right), & d_{32}=\left(E_{m}^{*^{\prime}}-E_{m 0}^{\prime}\right) B_{12}, & d_{44}=-d_{42}, \\
\gamma=\frac{1}{E_{m}^{*^{\prime 2}}}-\frac{1}{E_{m 0}^{\prime 2}}, & d_{42}=-\left(E_{m}^{*^{\prime}}-E_{m 0}^{\prime}\right) G_{12}, & d_{24}=V_{\infty} G_{31}, \\
d_{41}=-\left(E_{m}^{*^{\prime}} E_{q}^{*^{\prime}}-E_{m 0}^{\prime} E_{q 0}^{\prime}\right) B_{12}, & d_{31}=\left(E_{m}^{*^{\prime}} E_{q}^{*^{\prime}}-E_{m 0}^{\prime} E_{q 0}^{\prime}\right) G_{12} . & \\
\tilde{\phi}_{7}=\left[\begin{array}{llll}
c_{11} & c_{12} & c_{13} & c_{14}
\end{array}\right]\left[\begin{array}{cccc}
0 & 0 & d_{13} & d_{14} \\
0 & 0 & d_{23} & d_{24} \\
d_{31} & d_{32} & d_{33} & d_{34} \\
d_{41} & d_{42} & d_{43} & d_{44}
\end{array}\right]+\left[\begin{array}{llll}
0 & 0 & 0 & -2\left(E_{m}^{*^{\prime}}-E_{m 0}^{\prime}\right) G_{33}
\end{array}\right] .
\end{array}
$$

\section{Acknowledgments}

This work was supported by the Australian Research Council and the University of New South Wales at the Australian Defense Force Academy (UNSW@ADFA). 


\section{References}

[1] Taylor CW. Power System Voltage Stability. New York: McGraw-Hill; 1994.

[2] Vu KT, Liu CC, Taylor CW, Jimma KM. Voltage instability: mechanism and control strategies [power systems]. Proceedings of the IEEE 1995;83(11):1442-55.

[3] Overbye TJ. Effects of load modelling on analysis of power system voltage stability. IEEE Trans on Power Systems October 1994;16(5):329-38.

[4] Li Y, Chiang HD, Choi BK, Chen YT, Lauby DHHMG. Load models for modeling dynamic behaviors of reactive loads: Evaluation and comparison. International Journal of Electrical Power \& Energy Systems 2008;30(9):497-503.

[5] Hossain MJ, Pota HR, Ugrinovskii V. Short and long-term dynamic voltage instability. In: Proceedings of the 17th World Congress. Seoul, Korea; July, 2008, p. 9392-7.

[6] Cutsem TV, Vournas C. Voltage Stability of Electric Power Systems. Norwell, MA: Kluwer Academic; 1998.

[7] Williams BR, Schmus WR, Dawson DC. Transmission voltage recovery delayed by stalled air conditioners compressors. IEEE Trans on Power Systems 1992;7(3):1173-9.

[8] Leon JADD, Taylor CW. Understanding and solving short term voltage stability problems. In: IEEE Power Engineering Society Summer Meeting. Chicago, USA; July, 2002, p. $745-52$.

[9] Kundur P. Power System Stability and Control. New York: McGraw-Hill; 1994.

[10] Shaoyun G, Chung TS. Optimal active power flow incorporating FACTS devices with power flow control constraints. International Journal of Electrical Power \& Energy Systems 1998;20(5):321-4.

[11] Sanchez-Gasca JJ, Vittal V, Gibbard MJ, Messina AR, Vowles DJ, Liu S, et al. Inclusion of higher order terms for small-signal (modal) analysis: Committee report-task force on assessing the need to include higher order terms for small-signal (modal) analysis. IEEE Trans on Power Systems 2004;15(4):1306-12. 
[12] Martinez I, Messina A, Barocio E. Perturbation analysis of power systems: effects of second- and third-order nonlinear terms on system dynamic behaviour. Electric Power Systems Research, Elsevier 2004;71:159-67.

[13] Cao Y, Jiang L, Cheng S, Chen D, Malik O, Hope G. A nonlinear variable structure stabilizer for power system stability. IEEE Trans on Energy Conversion 1994;9(3):489-95.

[14] Lu Q, Sun YZ, Mei S. Nonlinear Control Systems and Power System Dynamics. Boston: Kluwer Academic Publishers; 2001.

[15] Seo JC, Kim TH, Park JK, Moon SI. An LQG based PSS design for controlling the SSR in power systems with series-compensated lines. IEEE Trans on Energy Conversion $1996 ; 11(2): 423-8$.

[16] Anderson BD, Moore JB. Optimal Control: Linear Quadratic Methods. Prentice-Hall; 1990.

[17] Ray LR. Stability robustness of uncertain LQG/LTR systems. IEEE Trans on Automatic control 1993;38(2):304-8.

[18] Kwang MS, Park JK. On the robust LQG control of TCSC for damping power system oscillations. IEEE Trans on Power Systems 2000;15(4):1306-12.

[19] Ferreira A, Barreiros JA, Jr. WB, de Souza JRB. A robust adaptive LQG/LTR TCSC controller applied to damp power system oscillations. Electric Power Systems Research $2007 ; 77: 956-64$.

[20] Dou CX, Zhang XZ, Guo SL, Mao CC. Delay-independent excitation control for uncertain large power systems using wide-area measurement signals. International Journal of Electrical Power \& Energy Systems 2010;32(3):210-7.

[21] Mielczarski W, Zajaczkowski AM. Nonlinear field voltage control of a synchronous generator using feedback linearisation. Automatica 1994;30(10):1625-30.

[22] Ramos RA, Alberto LFC, Bretas NG. Linear matrix inequality based controller design with feedback linearisation: Application to power systems. IEE Proceedings-Control Theory and Applications 2003;150(5):551-6. 
[23] Chapman JW, Ilic MD, King CA. Stabilizing a multi-machine power system via decentralized feedback linearizing excitation control. IEEE Trans on Energy Conversion $1993 ; 8(3): 830-8$.

[24] Xianrong C, Renzhou W, Yihan Y. The nonlinear excitation control of generators. In: IEEE Conference on Computer, Communication, Control and Power Engineering. Beijing, China; October, 1993, p. 23-6.

[25] Guo Y, Hill DJ, Wang Y. A robust decentralized excitation control of multimachine power systems. In: American Control Conference. San Diego, California, USA; June, 1999, p. 61-74.

[26] Gan D, Qu Z, Cai H. Multimachine power system excitation control design via theories of feedback linearisation control and nonlinear robust control. International Journal of System Science 2000;31(4):519-27.

[27] Qiu Z, Dorsey JF, Bond J, McCalley JD. Application of robust control to sustained oscillations in power systems. IEEE Trans on Circuits and Systems 1992;39(6):470-6.

[28] Hossain MJ, Pota HR, Ugrinovskii V, Ramos RA. Excitation control for large disturbances in power systems with dynamic loads. In: IEEE Power and Energy Society General Meeting. Calgary, Canada; July, 2009, p. 1-8.

[29] Petersen IR, Ugrinovskii VA, Savkin AV. Robust Control Design Using $H_{\infty}$ Methods. London: Springer; 2000.

[30] Lu YN. Electric Power System Dynamics. London: Academic Press; 1983.

[31] Bergen AR. Power System Analysis. New Jersey: Prentice-Hall; 1986.

[32] Leith DJ, Leithead WE. Survey of gain-scheduling analysis and design. International Journal of Control 2000;73(11):1001-25.

[33] Khalil HK. Nonlinear Systems. Macmillan, New York: Prentice-Hall; 1992.

[34] Ugrinovskii VA, Petersen IR. Minimax LQG control of stochastic partially observed uncertain systems. SIAM Journal of Control and Optimization 2001;40(4):1189-226. 